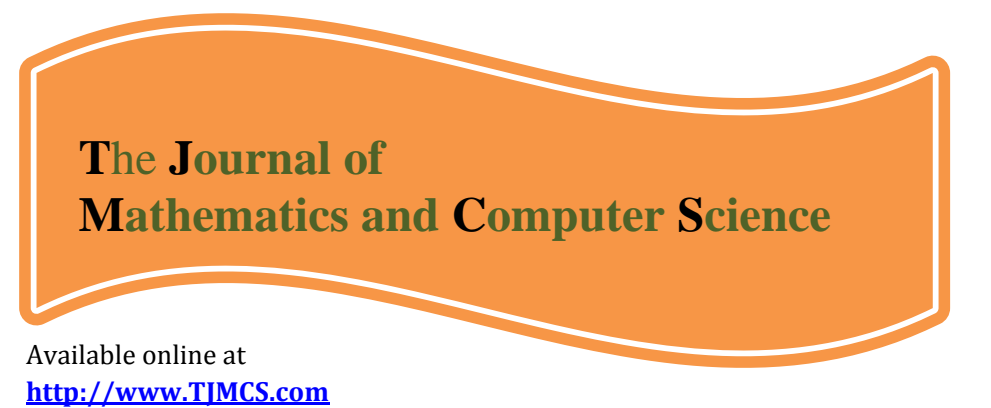

The Journal of Mathematics and Computer Science Vol .1 No.3 (2010) 174-186

\title{
COMPARISONS OF QUADRATURE SCHEMES WITH ARITHMETIC MEAN ITERATIVE METHOD FOR SECOND KIND LINEAR FREDHOLM INTEGRAL EQUATIONS
}

\author{
1,* Mohana Sundaram Muthuvalu, ${ }^{2}$ Jumat Sulaiman \\ 1,2 School of Science and Technology, Universiti Malaysia Sabah, \\ Locked Bag 2073, 88999 Kota Kinabalu, Sabah, Malaysia \\ 1 sundaram_at2@yahoo.com, \\ 2 jumat@ums.edu.my \\ * - Corresponding author
}

Received: December 2009, Revised: February 2010

Online Publication: August 2010

\begin{abstract}
In this paper, we investigate the applications of two different quadrature schemes that is repeated trapezoidal (RT) and repeated modified trapezoidal (RMT) schemes via Arithmetic Mean iterative method to solve second kind linear Fredholm integral equations. Furthermore, the derivation and implementation of the proposed method are also included. Numerical tests and comparisons are given to illustrate the effectiveness of the proposed method.
\end{abstract}

Keywords: Linear Fredholm equations, repeated trapezoidal, repeated modified trapezoidal, Arithmetic Mean

2000 Mathematics Subject Classification: 41A55, 45A05, 45B05, 65D32, 65F10

\section{INTRODUCTION}

Generally, second kind linear integral equations of Fredholm type in the generic form can be defined as follows 


$$
\lambda y(x)+\int_{\Gamma} K(x, t) y(t) d t=f(x), \Gamma=[a, b] \lambda \neq 0
$$

where the parameter $\lambda$, kernel $K \in L^{2}(\Gamma \times \Gamma)$ and free term $f \in L(\Gamma)$ are given, and $y \in L(\Gamma)$ is the unknown function to be determined. The kernel function $K(x, t)$ is assumed to be absolutely integrable and satisfy other properties that are sufficient to imply the Fredholm alternative theorem. Meanwhile, Eq. (1) also can be rewrite in the equivalent operator form

$$
(\lambda+\kappa) y=f .
$$

\section{Theorem (Fredholm Alternative) [3]}

Let $\chi$ be a Banach space and let $\kappa: \chi \rightarrow \chi$ be compact. Then the equation $(\lambda+\kappa) y=f, \lambda \neq 0$ has a unique solution $x \in \chi$ if and only if the homogeneous equation $(\lambda+\kappa) z=0$ has only the trivial solution $z=0$. In such a case, the operator $\lambda+\kappa: \chi \underset{\text { onto }}{\rightarrow} \chi$ has a bounded inverse $(\lambda+\kappa)^{-1}$.

\section{Definition (Compact operators) [3]}

Let $\chi$ and $\mathrm{Y}$ be normed vector space and let $\kappa: \chi \rightarrow \mathrm{Y}$ be linear. Then $\kappa$ is compact if the set $\{\kappa x \mid\|x\| x \leq 1\}$ has compact closure in $\mathrm{Y}$. This is equivalent to saying that for every bounded sequence $\left\{x_{n}\right\} \subset \chi$, the sequences $\left\{\kappa x_{n}\right\}$ has a subsequence that is convergent to some point in Y . Compact operators are also called completely continuous operators.

In many application areas, numerical approaches were used widely to solve Fredholm integral equations. By solving Eq. (2) numerically, we either seek to determine an approximation solution in a chosen finite dimensional space $V_{n}$ by a projection method $[5,6,8,11,14]$

$$
\left(\lambda+P_{n} \kappa\right) y_{n}=P_{n} f
$$

where $y_{n} \in V_{n}$ and $P_{n}: C \rightarrow V_{n}$ is a projection operator, or use the quadrature method

$$
\left(\lambda I+\kappa_{n}\right) y_{n}=f
$$

where $\kappa_{n}$ approximates $\kappa$ and is obtained by discretization of $\kappa$ by an $n$-point quadrature method; see $[9,10,12,13,18]$. Such discretizations of integral equations lead to dense linear systems and can be prohibitively expensive to solve as $n$, the order of the linear system of linear algebraic equations increases. Thus, iterative methods are the natural options for efficient solutions.

Consequently, two-stage iterative method also called as inner and outer iteration schemes have been proposed widely to be one of the feasible and successful classes of numerical algorithms for solving any linear system. Actually, there are many two-stage iterative methods can be considered such as the Alternating Group Explicit (AGE) [7], Iterative Alternating Decomposition Explicit (IADE) [19], Reduced Iterative Alternating Decomposition Explicit (RIADE) [20], Block Jacobi [2] and Arithmetic Mean (AM) [17] methods. However, in this paper, we examined the applications of the Arithmetic Mean method with two different quadrature methods in solving second kind linear Fredholm integral equations. 
The outline of this paper is organized in following way. In Section 2, the formulation of the quadrature approximation equations based on repeated trapezoidal (RT) and repeated modified trapezoidal (RMT) methods will be elaborated. The latter section of this paper will discuss the formulations of the AM method, and some numerical results will be shown in fourth section to assert the performance of the iterative methods. Finally, Section 5 contains some conclusions and directions of the future works.

\section{QUADRATURE APPROXIMATION EQUATIONS}

As explained in the previous section, a discretization scheme based on method of quadrature was used to construct an approximation equation of an integral equation by approximating the integral to finite sums. To facilitate in formulating the approximation equations for linear Fredholm equation of the second kind, further discussion will be restricted onto repeated trapezoidal (RT) and repeated modified trapezoidal (RMT) methods, which are based on interpolation formulas. In next subsections, application of the both schemes to discretize the Fredholm integral equations of the second kind will be explained.

\subsection{Repeated Trapezoidal}

The trapezoidal method is one of numerical integration methods derived by integrating the linear interpolation formula with equally spaced data points. Trapezoidal method for approximating definite integral $\int_{a}^{b} y(t) d t$ can be defined as follows

$$
\int_{a}^{b} y(t) d(t)=\frac{h}{2}(y(a)+y(b))+\varepsilon_{n}(y)
$$

and its repeated formula can be shown as

$$
\int_{a}^{b} y(t) d t=\frac{h}{2} y(a)+h \sum_{j=1}^{n-1} y\left(t_{j}\right)+\frac{h}{2} y(b)+\varepsilon_{n}(y)
$$

where the constant step size, $h$ is defined as

$$
h=\frac{b-a}{n} .
$$

$n, t_{j}$ and $\varepsilon_{n}(y)$ are the number of subintervals in the interval $[a, b]$, abscissas of the partition points of the integration interval $[a, b]$ and truncation error respectively. By applying Eq. (3) into Eq. (1) and neglecting the error, $\varepsilon_{n}(y)$ a system of linear algebraic equations can be formed for approximation values of $y(x)$ at the nodes $x_{0}, x_{1}, \ldots, x_{n}$. Therefore, the repeated trapezoidal approximation equations for Eq. (1) can be shown as follows

$$
\lambda y_{i}+\left(\frac{h}{2} K_{i, 0} y_{0}+h \sum_{j=1}^{n-1} K_{i, j} y_{j}+\frac{h}{2} K_{i, n} y_{n}\right)=f_{i}, i=0,1,2, \cdots, n
$$

Further discussions on RT method to solve Fredholm integral equations can be found in [3, 4].

\subsection{Repeated Modified Trapezoidal}


Besides trapezoidal method, definite integrals $\int_{a}^{b} y(t) d t$ can be also approximated by using the modified trapezoidal method, obtained based on the Hermite interpolation [21]. Hermite interpolation is a method closely related to the Newton divided difference interpolation that considers derivatives at data points. The formula for the method is defined as follows

$$
\int_{a}^{b} y(t) d t=\frac{h}{2}(y(a)+y(b))+\frac{h^{2}}{12}\left(y^{\prime}(a)+y^{\prime}(b)\right)+\varepsilon_{n}(y)
$$

Then, the RMT formula is as follows

$$
\int_{a}^{b} y(t) d t=\frac{h}{2} y(a)+h \sum_{j=1}^{n-1} y\left(t_{j}\right)+\frac{h}{2} y(b)+\frac{h^{2}}{12}\left(y^{\prime}(a)-y^{\prime}(b)\right)+\varepsilon_{n}(y)
$$

In order to discretize Eq. (1) using RMT method, conditions of $K(x, t)$ and $f(x)$ must be differentiable with respect to their variables should be satisfied. Meanwhile, two cases which are whether $\frac{\partial K(x, t)}{\partial x \partial t}$ exist or not, also need to be considered. Formulation for both cases explained in [18]. Before further clarification, the following notation will be used for simplicity.

$$
\begin{aligned}
J_{i, j} & =\frac{\partial K\left(x_{i}, t_{j}\right)}{\partial t_{j}} \\
H_{i, j} & =\frac{\partial K\left(x_{i}, t_{j}\right)}{\partial x_{i}} \\
L_{i, j} & =\frac{\partial K\left(x_{i}, t_{j}\right)}{\partial x_{i} \partial t_{j}} \\
y_{i}^{\prime} & =y^{\prime}\left(x_{i}\right) \\
f_{i}^{\prime} & =f^{\prime}\left(x_{i}\right)
\end{aligned}
$$

By applying the formula for RMT in Eq. (10) into Eq. (1), the approximation equations for both cases shown as follows

Case 1: The partial derivative $L_{i, j}=\frac{\partial K(x, t)}{\partial x \partial t}$ does not exists

$$
\begin{aligned}
& y_{i}+A_{i, 0} y_{0}+h \sum_{j=1}^{n-1} K_{i, j} y_{j}+B_{i, n} y_{n}+\frac{h^{2}}{12} K_{i, 0} y_{0}^{\prime}-\frac{h^{2}}{12} K_{i, n} y_{n}^{\prime}=f_{i}, \quad i=0,1, \cdots, n-1, n \\
& y_{0}^{\prime}+\frac{h}{2} H_{0,0} y_{0}+h \sum_{j=1}^{n-1} H_{0, j} y_{j}+\frac{h}{2} H_{0, n} y_{n}=f_{0}^{\prime} \\
& y_{n}^{\prime}+\frac{h}{2} H_{n, 0} y_{0}+h \sum_{j=1}^{n-1} H_{n, j} y_{j}+\frac{h}{2} H_{n, n} y_{n}=f_{n}^{\prime}
\end{aligned}
$$

Case 2: The partial derivative $L_{i, j}=\frac{\partial K(x, t)}{\partial x \partial t}$ exists 


$$
\left.\begin{array}{l}
y_{i}+A_{i, 0} y_{0}+h \sum_{j=1}^{n-1} K_{i, j} y_{j}+B_{i, n} y_{n}+\frac{h^{2}}{12} K_{i, 0} y_{0}^{\prime}-\frac{h^{2}}{12} K_{i, n} y_{n}^{\prime}=f_{i}, \quad i=0,1, \cdots, n-1, n \\
y_{0}^{\prime}+C_{0,0} y_{0}+h \sum_{j=1}^{n-1} H_{0, j} y_{j}+D_{0, n} y_{n}+\frac{h^{2}}{12} H_{0,0} y_{0}^{\prime}-\frac{h^{2}}{12} H_{0, n} y_{n}^{\prime}=f_{0}^{\prime} \\
y_{n}^{\prime}+C_{n, 0} y_{0}+h \sum_{j=1}^{n-1} H_{n, j} y_{j}+D_{n, n} y_{n}+\frac{h^{2}}{12} H_{n, 0} y_{0}^{\prime}-\frac{h^{2}}{12} H_{n, n} y_{n}^{\prime}=f_{n}^{\prime}
\end{array}\right\}
$$

where

$$
\begin{aligned}
A_{i, j} & =\frac{h}{2} K_{i, j}+\frac{h^{2}}{12} J_{i, j} \\
B_{i, j} & =\frac{h}{2} K_{i, j}-\frac{h^{2}}{12} J_{i, j} \\
C_{i, j} & =\frac{h}{2} H_{i, j}+\frac{h^{2}}{12} L_{i, j} \\
D_{i, j} & =\frac{h}{2} H_{i, j}-\frac{h^{2}}{12} L_{i, j}
\end{aligned}
$$

From Eqs. (5), (8) and (9), it is obvious that discretization of second kind Fredholm integral equations using RT or RMT method yields a system of linear equations as follows

$$
M \underset{\sim}{y}=f
$$

where the coefficient matrix $M$ is a dense matrix. Meanwhile, $f$ and $y$ are the right hand side vector and unknown vector to be determined respectively.

\section{FORMULATION OF ARITHMETIC MEAN METHOD}

As afore-mentioned, AM methods are one of the two-stage iterative methods and the iterative process involves of solving two independent systems such as $y^{1}$ and $y^{2}$. To develop the formulation of AM method, express the coefficient matrix $A$ as the matrix sum

$$
A=L+D+U
$$

where $L, D$ and $U$ are the strictly lower triangular, diagonal and strictly upper triangular matrices respectively. Thus, by adding positive acceleration parameter, $\omega$ the general scheme for AM method is defined by 


$$
\begin{aligned}
& (D+\omega L) \underset{\sim}{y^{1}}=((1-\omega) D-\omega U) \underset{\sim}{y^{(k)}+\omega f} \\
& (D+\omega U) \underset{\sim}{y^{2}}=((1-\omega) D-\omega L) \underset{\sim}{y^{(k)}+\omega f} \\
& y_{\sim}^{(k+1)}=\frac{1}{2}\left(\underset{\sim}{{ }_{\sim}^{1}}+\underset{\sim}{y^{2}}\right)
\end{aligned}
$$

where $y^{(0)}$ is an initial vector approximation to the solution and $0<\omega<2$.

The AM method requires a slight additional computational effort of the sum of two matrices at each iteration $k$, but its rate of convergence is relatively insensitive to the exact choice of the parameter $\omega$ [17]. Practically, the value of $\omega$ will be determined by implementing some computer programs and then choose one value of $\omega$, where its number of iterations is the smallest. The AM algorithm is explicitly performed by using all equations at level (1) and (2) alternatively until the specified convergence criterion is satisfied. By determining values of matrices $L, D$ and $U$ as stated in Eq. (14), the general algorithm for AM with RT and RMT methods to solve problem (1) would be generally described in Algorithms 1, 2 and 3. Generally, the basic idea for the convergence analysis of the AM method has been proved by [17].

\section{NUMERICAL EXPERIMENTS}

In order to compare the performances of the iterative methods described in the previous section, several numerical tests were carried out on the following both Fredholm integral equations.

\section{Example 1 [22]}

Consider the Fredholm integral equation of the second kind

$$
y(x)-\int_{0}^{1}\left(4 x t-x^{2}\right) y(t) d t=x, 0 \leq x \leq 1
$$

and the exact solution is given by

$$
y(x)=24 x-9 x^{2}
$$

\section{Example 2 [16]}

Consider the Fredholm integral equation of the second kind

$$
y(x)-\int_{0}^{1}\left(x^{2}+t^{2}\right) y(t) d t=x^{6}-5 x^{3}+x+10,0 \leq x \leq 1
$$

with the exact solution

$$
y(x)=x^{6}-5 x^{3}+\frac{1045}{28} x^{2}+x+\frac{2141}{84}
$$

For comparison, the Gauss-Seidel (GS) iterative method acts as control method of numerical results. Three criteria will be considered in comparison for GS with RT (GS-RT), GS with RMT (GS-RMT), AM with RT (AM-RT) and AM with RMT (AM-RMT) methods that is number of iterations, execution time and maximum absolute error. Throughout the simulations, 
the convergence test considered the tolerance error, $\varepsilon=10^{-10}$. In this paper, interval $[a, b]$ will be uniformly divided into $n=2^{m}, m \geq 2$ and discrete set of points be given as $x_{i}=a+i h$. Results of numerical simulations, which were obtained from implementations of the iterative methods for Examples 1 and 2, have been recorded in Tables 1 and 2 respectively. Meanwhile, reduction percentage of the execution time for the GS-RT, AM-RT and AM-RMT methods compared with GS-RMT method have been summarized in Table 3.

Table 1. Comparison of a number of iterations, execution time and maximum absolute error for the iterative methods at optimum value of $\omega$ (Example 1)

\begin{tabular}{|c|c|c|c|c|c|}
\hline \multirow{3}{*}{ Methods } & \multicolumn{5}{|c|}{ Number of iterations } \\
\hline & \multicolumn{5}{|c|}{$n$} \\
\hline & 512 & 1024 & 2048 & 4096 & 8192 \\
\hline GS-RT & 194 & 194 & 195 & 195 & 195 \\
\hline GS-RMT & 198 & 199 & 199 & 199 & 199 \\
\hline AM-RT & 84 & 84 & 84 & 84 & 84 \\
\hline \multirow[t]{2}{*}{ AM-RMT } & 46 & 46 & 46 & 46 & 46 \\
\hline & \multicolumn{5}{|c|}{ Execution time (seconds) } \\
\hline \multirow{2}{*}{ Methods } & \multicolumn{5}{|c|}{$n$} \\
\hline & 512 & 1024 & 2048 & 4096 & 8192 \\
\hline GS-RT & 2.62 & 10.77 & 38.77 & 145.01 & 570.58 \\
\hline GS-RMT & 2.91 & 14.02 & 48.83 & 177.01 & 644.35 \\
\hline AM-RT & 2.03 & 7.80 & 31.05 & 123.06 & 491.49 \\
\hline \multirow[t]{2}{*}{ AM-RMT } & 1.92 & 7.42 & 27.45 & 109.38 & 402.16 \\
\hline & \multicolumn{5}{|c|}{ Maximum absolute error } \\
\hline \multirow{2}{*}{ Methods } & \multicolumn{5}{|c|}{$n$} \\
\hline & 512 & 1024 & 2048 & 4096 & 8192 \\
\hline GS-RT & $4.6922 \mathrm{E}-4$ & $1.1730 \mathrm{E}-4$ & $2.9325 \mathrm{E}-5$ & 7.3307 E-6 & $1.8321 \mathrm{E}-6$ \\
\hline GS-RMT & $3.5954 \mathrm{E}-7$ & $4.5208 \mathrm{E}-8$ & $6.0171 \mathrm{E}-9$ & $1.1272 \mathrm{E}-9$ & $5.1823 \mathrm{E}-10$ \\
\hline AM-RT & $4.6922 \mathrm{E}-4$ & $1.1730 \mathrm{E}-4$ & $2.9325 \mathrm{E}-5$ & $7.3311 \mathrm{E}-6$ & $1.8326 \mathrm{E}-6$ \\
\hline AM-RMT & $3.5913 \mathrm{E}-7$ & $4.4840 \mathrm{E}-8$ & $5.6402 \mathrm{E}-9$ & $7.4613 \mathrm{E}-10$ & $1.3497 \mathrm{E}-10$ \\
\hline
\end{tabular}

Table 2. Comparison of a number of iterations, execution time and maximum absolute error for the iterative methods at optimum value of $\omega$ (Example 2)

\begin{tabular}{cccccc}
\hline \multicolumn{5}{c}{ Number of iterations } \\
\hline \multirow{2}{*}{ Methods } & $\mathbf{5 1 2}$ & $\mathbf{1 0 2 4}$ & $\mathbf{2 0 4 8}$ & $\mathbf{4 0 9 6}$ & $\mathbf{8 1 9 2}$ \\
\cline { 2 - 6 } & 56 & 56 & 56 & 56 & 56 \\
GS-RT & 57 & 57 & 57 & 57 & 57 \\
GS-RMT & 32 & 32 & 32 & 32 & 32 \\
\hdashline AM-RT & 25 & 26 & 26 & 26 & 26 \\
AM-RMT & \multicolumn{5}{c}{ Execution time (seconds) } \\
\hline \multicolumn{6}{c}{}
\end{tabular}




\begin{tabular}{cccccc}
\hline \multirow{2}{*}{ Methods } & \multicolumn{5}{c}{$\boldsymbol{n}$} \\
\cline { 2 - 6 } & $\mathbf{5 1 2}$ & $\mathbf{1 0 2 4}$ & $\mathbf{2 0 4 8}$ & $\mathbf{4 0 9 6}$ & $\mathbf{8 1 9 2}$ \\
\hline GS-RT & 0.89 & 3.47 & 17.06 & 55.85 & 189.98 \\
GS-RMT & 0.95 & 3.96 & 20.44 & 70.89 & 242.87 \\
\hdashline AM-RT & 0.61 & 2.56 & 9.39 & 38.17 & 167.44 \\
AM-RMT & 0.55 & 2.45 & 9.06 & 36.88 & 155.23 \\
\hline \multicolumn{6}{c}{ Maximum absolute error } \\
\hline \multirow{2}{*}{ Methods } & $\mathbf{5 1 2}$ & $\mathbf{n}$ \\
\cline { 2 - 6 } & $\mathbf{1 0 2 4}$ & $\mathbf{2 0 4 8}$ \\
\hline GS-RT & $4.7770 \mathrm{E}-4$ & $1.1942 \mathrm{E}-4$ & $2.9856 \mathrm{E}-5$ & $7.4639 \mathrm{E}-6$ & $1.8659 \mathrm{E}-6$ \\
GS-RMT & $8.6136 \mathrm{E}-7$ & $1.0751 \mathrm{E}-7$ & $1.3495 \mathrm{E}-8$ & $1.7567 \mathrm{E}-9$ & $2.9065 \mathrm{E}-10$ \\
\hdashline AM-RT & $4.7770 \mathrm{E}-4$ & $1.1942 \mathrm{E}-4$ & $2.9856 \mathrm{E}-5$ & $7.4639 \mathrm{E}-6$ & $1.8659 \mathrm{E}-6$ \\
AM-RMT & $8.6130 \mathrm{E}-7$ & $1.0744 \mathrm{E}-7$ & $1.3422 \mathrm{E}-8$ & $1.6832 \mathrm{E}-9$ & $2.1709 \mathrm{E}-10$ \\
\hline
\end{tabular}

Table 3. Reduction percentage of the execution time for the GS-RT, AM-RT and AM-RMT methods compared with GS-RMT method

\begin{tabular}{ccc}
\hline Methods & Example 1 & Example 2 \\
\hline GS-RT & $9.96-23.19 \%$ & $6.31-21.78 \%$ \\
AM-RT & $23.72-44.37 \%$ & $31.05-54.07 \%$ \\
AM-RMT & $34.02-47.08 \%$ & $36.08-55.68 \%$ \\
\hline
\end{tabular}

\section{CONCLUSIONS}

In the previous section, it has shown that the quadrature approximation equations based on repeated trapezoidal and repeated modified trapezoidal methods can easily generate a system of linear equations. From the Eqs. (8), (11) and (12), it can be found that approximation equations based on RT method involve $(n+1)$ equations and $(n+3)$ equations for RMT method. Through numerical results obtained in Tables 1 and 2, it clearly shows that by applying the AM method can reduce number of iterations compared to the GS method for both discretization methods. In terms of execution time, GS-RT, AM-RT and AM-RMT methods are faster compared to GS-RMT method (refer Table 3). Through the observation from the results obtained, it shows that RMT method is more accurate than the RT method. Overall, the numerical results have shown that the AM-RMT method is more superior compared to the GS-RT, GS-RMT and AM-RT methods. For future works, this study will be extended to investigate the applications of the complexity reduction techniques $[1,15]$ in solving Fredholm integral equations.

\section{ACKNOWLEDGMENT}

The authors acknowledge the Postgraduate Research Grant, Universiti Malaysia Sabah (GPS0003-SG-1/2009) for the completion of this article. 


\section{REFERENCES}

[1] Abdullah, A.R. (1991). The four point Explicit Decoupled Group (EDG) method: A fast Poisson solver. International Journal Computer Mathematics 38, 61-70.

[2] Allahviranloo, T., Ahmady, E., Ahmady, N., Alketaby, K.S. (2006). Block Jacobi two-stage method with Gauss-Sidel inner iterations for fuzzy system of linear equations. Applied Mathematics and Computation 175, 1217-1228.

[3] Atkinson, K.E. (1997). The Numerical Solution of Integral Equations of the Second Kind. Cambridge University Press, United Kingdom.

[4] Baker, C.T.H. (1977). The Numerical Treatment of Integral Equations. Clarendon Press, Oxford.

[5] Chen, Z., Micchelli, C.A., Xu, Y. (2002). Fast collocation methods for second kind integral equations. SIAM Journal on Numerical Analysis 40(1), 344-375.

[6] Chen, Z., Wu, B., Xu, Y. (2007). Fast numerical collocation solutions of integral equations. Communications on Pure and Applied Analysis 6(3), 643-666.

[7] Evans, D.J., Sahimi, M.S. (1988). The Alternating Group Explicit iterative method (AGE) to solve parabolic and hyperbolic partial differential equations. Ann. Rev. Num. Fluid Mech. and Heat Trans. 2, 283-389.

[8] Kaneko, H. (1989). A projection method for solving Fredholm integral equations of the second kind. Applied Numerical Mathematics 5(4), 333-344.

[9] Laurie, D.P. (2001). Computation of Gauss-type quadrature formulas. Journal of Computational and Applied Mathematics 127, 201-217.

[10] Lin, F.-R. (2003). Preconditioned iterative methods for the numerical solution of Fredholm equations of the second kind. Calcolo 40, 231-248.

[11] Maleknejad, K., Kajani, M.T. (2003). Solving second kind integral equations by Galerkin methods with hybrid Legendre and Block-Pulse functions. Applied Mathematics and Computation 145, 623-629.

[12] Muthuvalu, M.S., Sulaiman, J. (2008). Half-Sweep Geometric Mean method for solution of linear Fredholm equations. Matematika 24(1), 75-84.

[13] Muthuvalu, M.S., Sulaiman, J. (2009). Half-Sweep Arithmetic Mean method with high-order Newton-Cotes quadrature schemes to solve linear second kind Fredholm equations. Journal of Fundamental Sciences 5(1), 7-16.

[14] Oladejo, S.O., Mojeed, T.A., Olurode, K.A. (2008). The application of cubic spline collocation to the solution of integral equations. Journal of Applied Sciences Research 4(6), 748-753.

[15] Othman, M., Abdullah, A.R. (2000). An efficient Four Point Modified Explicit Group Poisson solver. International Journal of Computer Mathematics 76, 203-217.

[16] Polyanin, A.D., Manzhirov, A.V. (1998). Handbook of Integral Equations. CRC Press LLC, Florida.

[17] Ruggiero, V., Galligani, E. (1990). An iterative method for large sparse systems on a vector computer. Computers and Mathematics with Applications 20(1), 25-28. 
[18] Saberi-Nadjafi, J., Heidari, M. (2007). Solving linear integral equations of the second kind with repeated modified trapezoid quadrature method. Applied Mathematics and Computation 189, 980-985.

[19] Sahimi, M.S., Ahmad, A., Bakar, A.A. (1993). The Iterative Alternating Decomposition Explicit (IADE) method to solve the heat conduction equation. International Journal of Computer Mathematics 47, 219-229.

[20] Sahimi, M.S., Khatim, M. (2001). The Reduced Iterative Alternating Decomposition Explicit (RIADE) method for diffusion equation. Pertanika Journal of Science and Technology 9(1), 1320.

[21] Stoer, J., Bulirsch, R. (1993). Introduction to Numerical Analysis. Springer-Verlag.

[22] Wang, W. (2006). A new mechanical algorithm for solving the second kind of Fredholm integral equation. Applied Mathematics and Computation 172, 946-962.

\section{Algorithm 1. AM with RT method}

i) Level (1)

Calculate

$$
y_{i}^{1} \leftarrow \begin{cases}\left((1-\omega)\left(1+\frac{h}{2} K_{i, i}\right) y_{i}{ }^{(k)}-\omega h \sum_{j=1}^{n-1} K_{i, j} y_{j}{ }^{(k)}-\frac{\omega h}{2} K_{i, n} y_{n}{ }^{(k)}+\omega f_{i}\right) /\left(1+\frac{h}{2} K_{i, i}\right) & , i=0 \\ \left((1-\omega)\left(1+\frac{h}{2} K_{i, i}\right) y_{i}{ }^{(k)}-\frac{\omega h}{2} K_{i, 0} y_{0}{ }^{(k+1)}-\omega h \sum_{j=1}^{n-1} K_{i, j} y_{j}{ }^{(k+1)}+\omega f_{i}\right) /\left(1+\frac{h}{2} K_{i, i}\right) & , i=n \\ \left((1-\omega)\left(1+h K_{i, i}\right) y_{i}{ }^{(k)}-\frac{\omega h}{2} K_{i, 0} y_{0}{ }^{(k+1)}-\omega h \sum_{j=1}^{i-1} K_{i, j} y_{j}{ }^{(k+1)}-\omega h \sum_{j=i+1}^{n-1} K_{i, j} y_{j}{ }^{(k)}-\frac{\omega h}{2} K_{i, n} y_{n}{ }^{(k)}+\omega f_{i}\right) /\left(1+h K_{i, i}\right) & , i=1, \cdots, n-1\end{cases}
$$

ii) Level (2)

Calculate

$$
y_{i}^{2} \leftarrow \begin{cases}\left((1-\omega)\left(1+\frac{h}{2} K_{i, i}\right) y_{i}{ }^{(k)}-\omega h \sum_{j=1}^{n-1} K_{i, j} y_{j}{ }^{(k+1)}-\frac{\omega h}{2} K_{i, n} y_{n}{ }^{(k+1)}+\omega f_{i}\right) /\left(1+\frac{h}{2} K_{i, i}\right) & , i=0 \\ \left((1-\omega)\left(1+\frac{h}{2} K_{i, i}\right) y_{i}{ }^{(k)}-\frac{\omega h}{2} K_{i, 0} y_{0}{ }^{(k)}-\omega h \sum_{j=1}^{n-1} K_{i, j} y_{j}{ }^{(k)}+\omega f_{i}\right) /\left(1+\frac{h}{2} K_{i, i}\right) & , i=n \\ \left((1-\omega)\left(1+h K_{i, i}\right) y_{i}{ }^{(k)}-\frac{\omega h}{2} K_{i, 0} y_{0}{ }^{(k)}-\omega h \sum_{j=1}^{i-1} K_{i, j} y_{j}{ }^{(k)}-\omega h \sum_{j=i+1}^{n-1} K_{i, j} y_{j}{ }^{(k+1)}-\frac{\omega h}{2} K_{i, n} y_{n}{ }^{(k+1)}+\omega f_{i}\right) /\left(1+h K_{i, i}\right) & , i=1, \cdots, n-1\end{cases}
$$

iii)For $i=0,1, \cdots, n-1, n$

Calculate

$$
y_{i}^{(k+1)} \leftarrow \frac{1}{2}\left(y_{i}^{1}+y_{i}^{2}\right)
$$


Algorithm 2. AM with RMT (Case 1) method

i) Level (1)

Calculate

$$
\begin{aligned}
& \left\{\begin{array}{l}
(1-\omega)\left(1+A_{i, i}\right) y_{i}{ }^{(k)}-\omega h \sum_{j=1}^{n-1} K_{i, j} y_{j}{ }^{(k)}-\omega B_{i, n} y_{n}{ }^{(k)}-\frac{\omega h^{2}}{12} K_{i, 0} y_{0}^{(k)} \\
+\frac{\omega h^{2}}{12} K_{i, n} y_{n}^{\left({ }^{(k)}\right.}+\omega f_{i}
\end{array}\right) /\left(1+A_{i, i}\right) \quad, i=0 \\
& \left(\begin{array}{l}
(1-\omega)\left(1+B_{i, i}\right) y_{i}^{(k)}-\omega A_{i, 0} y_{n}{ }^{(k+1)}-\omega h \sum_{j=1}^{n-1} K_{i, j} y_{j}{ }^{(k+1)}-\frac{\omega h^{2}}{12} K_{i, 0} y_{0}^{(k)} \\
+\frac{\omega h^{2}}{12} K_{i, n} y_{n}^{(k)}+\omega f_{i}
\end{array}\right) /\left(1+B_{i, i}\right) \quad, i=n \\
& y_{i}^{1} \leftarrow\left\{\begin{array}{l}
(1-\omega)\left(1+h K_{i, i}\right) y_{i}{ }^{\left({ }^{2}\right)}-\omega A_{i, 0} y_{n}{ }^{(k+1)}-\omega h \sum_{j=1}^{i-1} K_{i, j} y_{j}{ }^{(k+1)}-\omega h \sum_{j=i+1}^{n-1} K_{i, j} y_{j}{ }^{(k)} \\
-\omega B_{i, n} y_{n}{ }^{(k)}-\frac{\omega h^{2}}{12} K_{i, 0} y_{0}{ }^{\left({ }^{(k)}\right.}+\frac{\omega h^{2}}{12} K_{i, n} y_{n}{ }^{(k)}+\omega f_{i}
\end{array}\right) /\left(1+h K_{i, i}\right) \quad, i=1, \cdots, n-1 \\
& (1-\omega) y_{0}{ }^{(k)}-\frac{\omega h}{2} H_{0,0} y_{0}{ }^{(k+1)}-\omega h \sum_{j=1}^{n-1} H_{0, j} y_{j}{ }^{(k+1)}-\frac{\omega h}{2} H_{0, n} y_{n}{ }^{(k+1)}+\omega f_{0}^{\prime} \\
& (1-\omega) y_{n}{ }^{(k)}-\frac{\omega h}{2} H_{n, 0} y_{0}{ }^{(k+1)}-\omega h \sum_{j=1}^{n-1} H_{n, j} y_{j}{ }^{(k+1)}-\frac{\omega h}{2} H_{n, n} y_{n}{ }^{(k+1)}+\omega f_{n}^{\prime}
\end{aligned}
$$

ii) Level (2)

Calculate

$$
\begin{aligned}
& \left(\begin{array}{l}
(1-\omega)\left(1+A_{i, i}\right) y_{i}^{(k)}-\omega h \sum_{j=1}^{n-1} K_{i, j} y_{j}^{(k+1)}-\omega B_{i, n} y_{n}{ }^{(k+1)}-\frac{\omega h^{2}}{12} K_{i, 0} y_{0}^{(k+1)} \\
+\frac{\omega h^{2}}{12} K_{i, n} y_{n}^{{ }^{(k+1)}}+\omega f_{i}
\end{array}\right) /\left(1+A_{i, i}\right) \quad, i=0 \\
& \left(\begin{array}{l}
(1-\omega)\left(1+B_{i, i}\right) y_{i}^{\left({ }^{(k)}\right.}-\omega A_{i, 0} y_{n}{ }^{\left({ }^{k}\right)}-\omega h \sum_{j=1}^{n-1} K_{i, j} y_{j}^{(k)}-\frac{\omega h^{2}}{12} K_{i, 0} y_{0}^{(k+1)} \\
+\frac{\omega h^{2}}{12} K_{i, n} y_{n}^{(k+1)}+\omega f_{i}
\end{array}\right) /\left(1+B_{i, i}\right) \quad, i=n \\
& y_{i}^{2} \leftarrow\left\{\begin{array}{l}
(1-\omega)\left(1+h K_{i, i}\right) y_{i}{ }^{(k)}-\omega A_{i, 0} y_{n}{ }^{(k)}-\omega h \sum_{j=1}^{i-1} K_{i, j} y_{j}{ }^{(k)}-\omega h \sum_{j=i+1}^{n-1} K_{i, j} y_{j}{ }^{(k+1)} \\
-\omega B_{i, n} y_{n}{ }^{(k+1)}-\frac{\omega h^{2}}{12} K_{i, 0} y_{0}{ }^{(k+1)}+\frac{\omega h^{2}}{12} K_{i, n} y_{n}{ }^{(k+1)}+\omega f_{i}
\end{array}\right) /\left(1+h K_{i, i}\right) \quad, i=1, \cdots, n-1 \\
& (1-\omega) y_{0}{ }^{(k)}-\frac{\omega h}{2} H_{0,0} y_{0}{ }^{\left({ }^{k}\right)}-\omega h \sum_{j=1}^{n-1} H_{0, j} y_{j}{ }^{(k)}-\frac{\omega h}{2} H_{0, n} y_{n}{ }^{(k)}+\omega f_{0}^{\prime} \\
& (1-\omega) y_{n}{ }^{(k)}-\frac{\omega h}{2} H_{n, 0} y_{0}{ }^{\left({ }^{(k)}\right.}-\omega h \sum_{j=1}^{n-1} H_{n, j} y_{j}{ }^{(k)}-\frac{\omega h}{2} H_{n, n} y_{n}{ }^{\left({ }^{k}\right)}+\omega f_{n}^{\prime}
\end{aligned}
$$


iii)For $i=0,1, \cdots, n-1, n$

Calculate

$$
y_{i}^{(k+1)} \leftarrow \frac{1}{2}\left(y_{i}^{1}+y_{i}^{2}\right)
$$

Algorithm 3. AM with RMT (Case 2) method

i) Level (1)

Calculate

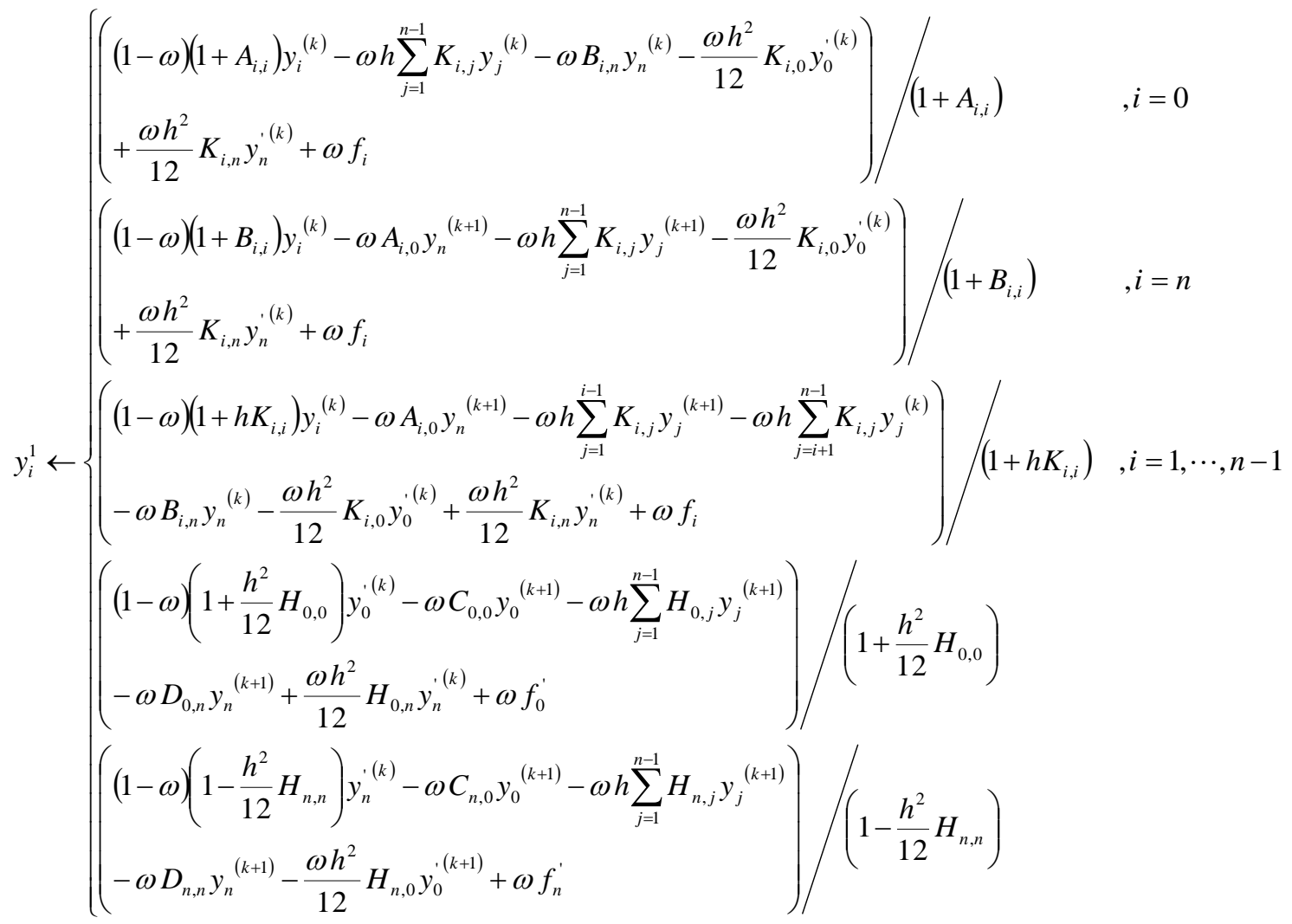

ii) Level (2)

Calculate 


$$
\begin{aligned}
& \left(\begin{array}{l}
(1-\omega)\left(1+A_{i, i}\right) y_{i}{ }^{(k)}-\omega h \sum_{j=1}^{n-1} K_{i, j} y_{j}{ }^{(k+1)}-\omega B_{i, n} y_{n}{ }^{(k+1)}-\frac{\omega h^{2}}{12} K_{i, 0} y_{0}^{(k+1)} \\
+\frac{\omega h^{2}}{12} K_{i, n} y_{n}^{(k+1)}+\omega f_{i}
\end{array}\right) /\left(1+A_{i, i}\right) \quad, i=0 \\
& \left(\begin{array}{l}
(1-\omega)\left(1+B_{i, i}\right) y_{i}^{(k)}-\omega A_{i, 0} y_{n}{ }^{(k)}-\omega h \sum_{j=1}^{n-1} K_{i, j} y_{j}{ }^{(k)}-\frac{\omega h^{2}}{12} K_{i, 0} y_{0}^{(k+1)} \\
+\frac{\omega h^{2}}{12} K_{i, n} y_{n}^{\left({ }^{(k+1)}\right.}+\omega f_{i}
\end{array}\right) /\left(1+B_{i, i}\right) \quad, i=n \\
& y_{i}^{2} \leftarrow\left\{\begin{array}{l}
(1-\omega)\left(1+h K_{i, i}\right) y_{i}{ }^{(k)}-\omega A_{i, 0} y_{n}{ }^{\left({ }^{2}\right)}-\omega h \sum_{j=1}^{i-1} K_{i, j} y_{j}{ }^{(k)}-\omega h \sum_{j=i+1}^{n-1} K_{i, j} y_{j}{ }^{(k+1)} \\
-\omega B_{i, n} y_{n}{ }^{(k+1)}-\frac{\omega h^{2}}{12} K_{i, 0} y_{0}{ }^{(k+1)}+\frac{\omega h^{2}}{12} K_{i, n} y_{n}{ }^{(k+1)}+\omega f_{i}
\end{array}\right) /\left(1+h K_{i, i}\right) \quad, i=1, \cdots, n-1 \\
& \left(\begin{array}{l}
(1-\omega)\left(1+\frac{h^{2}}{12} H_{0,0}\right) y_{0}^{(k)}-\omega C_{0,0} y_{0}{ }^{(k)}-\omega h \sum_{j=1}^{n-1} H_{0, j} y_{j}{ }^{(k)} \\
-\omega D_{0, n} y_{n}{ }^{(k)}+\frac{\omega h^{2}}{12} H_{0, n} y_{n}{ }^{(k+1)}+\omega f_{0}^{\prime}
\end{array}\right) /\left(1+\frac{h^{2}}{12} H_{0,0}\right) \\
& \left(\begin{array}{l}
(1-\omega)\left(1-\frac{h^{2}}{12} H_{n, n}\right) y_{n}{ }^{(k)}-\omega C_{n, 0} y_{0}{ }^{(k)}-\omega h \sum_{j=1}^{n-1} H_{n, j} y_{j}{ }^{(k)} \\
-\omega D_{n, n} y_{n}{ }^{(k)}-\frac{\omega h^{2}}{12} H_{n, 0} y_{0}{ }^{\left({ }^{(k)}\right.}+\omega f_{n}^{\prime}
\end{array}\right) /\left(1-\frac{h^{2}}{12} H_{n, n}\right)
\end{aligned}
$$

iii)For $i=0,1, \cdots, n-1, n$

Calculate

$$
y_{i}^{(k+1)} \leftarrow \frac{1}{2}\left(y_{i}{ }^{1}+y_{i}^{2}\right)
$$

